\title{
Grain Growth and Grain Translation in Crystals
}

\author{
Kevin McReynolds ${ }^{1}$, Kuo-An $\mathrm{Wu}^{2}$, and Peter Voorhees ${ }^{1}$ \\ Department of Materials Science and Engineering, Northwestern University, Evanston,
} IL 60208, USA

Department of Physics, National Tsing Hua University, Hsinchu 30013, Taiwan, Republic of China

\begin{abstract}
Grain growth is generally driven to minimize the overall grain boundary energy. However, for low-angle grain boundaries the requirement that lattice planes be continuous across the boundary gives rise to a coupling between the normal motion of the grain boundary and the tangential motion of the lattice. We show through phase-field crystal simulations this coupling in polycrystalline systems can give rise to a rigid body translation of the lattice as a grain shrinks. The process is mediated by significant climb of the dislocations in the boundary and dislocation reactions at the trijunctions. Thus the grain growth process is coupled to vacancy diffusion processes as well as the dynamics of grain trijunctions. Moreover, grain shrinkage can cease because of dislocation behavior near the trijunction, illustrating that this coupling can have an influence on the grain growth process in polycrystals.
\end{abstract}

Keywords: Grain boundary motion, Grain growth, Phase field, Coupling 


\section{Introduction}

Systems composed of domains of differing crystallographic orientation occur in systems ranging from dusty plasmas to colloidal crystals and polycrystalline materials [1,2]. Grain boundaries are classically understood to move in a way that reduces the total interfacial energy, in a manner similar to soap froths. However, unlike soap froths there can be a coupling between the normal motion of the grain boundary (grain growth) and the tangential motion of the lattice[3-15]. For example, low angle grain boundaries contain a unique constraint that lattice planes must be continuous across the grain boundary except at the dislocations that lie in the boundary. This induces a tangential velocity of the lattice that is proportional to the normal velocity of the grain boundary by the coupling parameter $\beta$, which is equal to the misorientation in the small angle limit. This coupling can either induce a stress during motion, or allow the boundary motion to couple to an applied stress. However, this coupling is surprisingly general, since it also occurs at high angle grain boundaries[4,9]. For a circular grain embedded in a single grain, this gives rise to a rotation of the lattice as the grain shrinks[4,16-21]. However, the effects of the coupling in the more general and widely observed case where there are multiple grains and grain boundary trijunctions has not been investigated.

We study the effect of coupling on a system with a circular grain embedded in a symmetric tilt planar grain boundary using the phase-field crystal (PFC) model. The PFC model is uniquely suited to studying nanoscale grain growth because it captures atomic motion over diffusive timescales. Atomic resolution is required to 
resolve the lattice continuity across the grain boundary and, as we will show, the diffusive timescales are necessary to observe grain growth. The PFC model has been previously used to study phenomena in solid state grain growth. Dislocations form spontaneously in the PFC model to relieve strain (e.g. at low-angle grain boundaries) and move through the crystal via climb and glide mechanisms [22,23]. For low-angle grain boundaries, the grain boundary energy follows the ReadShockley relationship[16,24,25]. The Asaro-Tiller-Grinfeld instability was studied using the PFC model, and the results were in quantitative agreement with continuum theory[26,27]. The PFC model has also been used to study coupling during grain growth[16,17,28-30], and the results match well with molecular dynamics simulations[9].

For this study, the symmetric tilt geometry was chosen as the worst case scenario for grain rotation according to Cahn and Taylor[3] where the rotation induced by the first matrix grain would be exactly opposed by the rotation induced by the second matrix grain, and grain growth would be arrested due to this geometric frustration.We find that the coupling of the normal grain boundary motion and lattice translation leads to grain growth that is markedly different from that of a simple soap froth. Furthermore, in contrast to the case of an isolated grain, when a grain is embedded at a symmetric planar tilt grain boundary the coupling between the normal motion of the boundary and the tangential motion of the lattice gives rise to a rigid body translation of the lattice without rotation as the grain shrinks. The process is mediated by significant climb of the dislocations in the boundary, and 
dislocation interactions at the trijunctions. Since climb is involved, this mechanism for grain growth depends on the self-diffusion process in the bulk crystal. Moreover, we find that grain shrinkage ceases if certain dislocation reactions do not occur at the trijunctions.

\section{The Phase-Field Crystal Model}

We use the dimensionless PFC model that employs the free energy functional $[24,25]$,

$$
\mathcal{F}=\int d \vec{r}\left\{\psi\left[-\epsilon+\left(1+\nabla^{2}\right)^{2}\right] \frac{\psi}{2}+\frac{\psi^{4}}{4}\right\}
$$

where $\mathcal{F}$ is the dimensionless free-energy density, $\psi$ is the dimensionless atomic density field defined as time-averaged density of atoms relative to a reference value, and $\epsilon$ is a temperature parameter.The evolution of $\psi$ follows the standard phasefield evolution for a conserved order parameter, with $t$ as the dimensionless time

$$
\frac{\partial \psi}{\partial t}=\nabla^{2} \frac{\delta \mathcal{F}}{\delta \psi}=\nabla^{2}\left[-\epsilon \psi+\left(1+\nabla^{2}\right)^{2} \psi+\psi^{3}\right]
$$

where the equations have been nondimensionalized by the length scale given by the lattice spacing and time scale related to the lattice spacing and diffusion coefficient $[25,31]$. The PFC model has roots in classical density functional theory (CDFT). From CDFT the order parameter $\psi$ can be viewed as a dimensionless timeaveraged atomic density field. Peaks in $\psi$ can be considered as the positions of the atoms. However, these peaks also occur at lattice sites. As the height of a peak decreases, the vacancy concentration at that site increases until the site disappears. Thus while the average density of the system is conserved, the number of peaks (or 
lattice sites) in the system is not, since lattice sites can be created and destroyed by physical mechanisms such as dislocation climb. The simplicity of the PFC model allows us to identify the system-independent mechanisms responsible for grain growth in polycrystalline systems.

\section{Results and Discussion}

\subsection{System Description}

The PFC model, Eq. (2), was solved with periodic boundary conditions using $\epsilon=$ 0.1(a relatively high temperature, close to the solid-liquid phase boundary) unless otherwise stated, the average density $\bar{\psi}=-0.195$, and a hexagonal twodimensional lattice. The initial density profile is given by the single-mode approximation, which then relaxes over short timescales. The three-grain system consists of circular grain embedded in a symmetric tilt planar grain boundary with a misorientation of $\pm \theta$ between the embedded grain and each of the two outer grains, and a misorientation $2 \theta$ of between the two outer grains (Fig. 1). The initial diameter of the embedded grain was set as not more than $60 \%$ of the smallest dimension of the rectangular computational domain. The values of $\theta$ are limited due to the periodic boundary conditions. Misorientations of $3.4^{\circ}, 5.2^{\circ}$, and $7.1^{\circ}$ were chosen using the method of Mellenthin et al.[32]. The $5.2^{\circ}$ system was tested using square meshes with $768^{2}, 1152^{2}$, and $2304^{2}$ mesh points. The mesh spacing was held constant yielding three different system sizes with three different initial sizes of the embedded grain. The mesh spacing varied slightly between the $\mathrm{x}$ and $\mathrm{y}$ directions because of the periodicity condition but was held constant between 
system sizes. The $3.4^{\circ}$ and $7.1^{\circ}$ systems were computed using the $1152^{2}$ system size. The mesh spacing varies slightly between the three different misorientations to maintain periodicity but is kept less than $\sqrt{3} / 16$, which is required for numerical accuracy. All distances in this paper are in units of the one-mode lattice parameter, but the system size was set using the equilibrium lattice parameter corresponding the free-energy minimum.

Fig.1 (color online). An example of the density field near the beginning of the simulation for the smallest system size. The lighter areas, those with low density, are where the periodicity of the lattice is interrupted by dislocations. There are peaks in the density field at each lattice point. The black lines indicate the path of the dislocations as the grain shrinks.

\subsection{Grain Translation}

As the embedded grain shrinks, the dislocations move towards the plane of the planar bicrystal grain boundary in a path that is approximately perpendicular to it (Fig. 1). This maintains the constant average spacing of the dislocations, indicating that the misorientation does not change and therefore no rotation occurs. However, we find that there is rigid body tangential translation of the embedded grain (in the $-x$ direction), and that this motion exists in all of the initial conditions employed at $\epsilon=0.1$. The translation was significant: over four lattice parameters in the largest system.

If this translation is the result of the coupling described in Section 1, the coupling parameter follows from the slope of the displacement of the embedded grain's lattice (tangential motion) vs. grain size (normal motion) curve and should be equal to the misorientation between the embedded grain and the outer grains. The grain 
size was measured as half the largest distance between the embedded grain boundaries in a direction perpendicular to the outer planar grain boundaries. Since the motion of the boundary is relatively uniform away from the trijunctions, this captures the normal motion of the entire grain boundary. The rigid body displacement was measured by tracking the location of a density peak in the center of the embedded grain. The relationship between the grain size and displacement is linear, but the coupling parameters are about 5-10\% lower than the theoretical values. This difference is a result of a small, fraction of a lattice parameter, movement of the outer grains ${ }^{1}$. Since the coupling is based on the continuity of the lattice planes, the movement of the outer grains constitutes a moving reference frame for the embedded grain. Adding this correction gives coupling parameters that are about $1-6 \%$ higher than the theoretical values as shown in Table 1. This good agreement with theory supports the notion that the movement of the embedded grain's lattice is due to the geometrically necessary coupling between the normal motion of the grain boundary and the tangential motion of the lattice.

Table 1. Calculated values of the coupling parameter. ${ }^{*}$ These two simulations have different initial dislocation structures in the boundary.

\begin{tabular}{ccc}
\hline $\begin{array}{c}\text { Misorientation } \\
(\boldsymbol{\theta})\end{array}$ & $\begin{array}{c}\text { Initial } \\
\text { Radius }\end{array}$ & $\begin{array}{c}\boldsymbol{\beta}_{\text {simulation }} \\
/ \boldsymbol{\beta}_{\text {theory }}\end{array}$ \\
\hline $3.42^{\circ}$ & 30.2 & 1.033 \\
$5.21^{\circ}$ & 19.9 & 1.043 \\
$5.21^{\circ}$ & $29.8^{*}$ & 1.063 \\
$5.21^{\circ}$ & $29.8^{*}$ & 1.044 \\
$5.21^{\circ}$ & 59.5 & 1.015
\end{tabular}

${ }^{1}$ This motion is primarily in response to the dislocation reactions at the trijunctions (and to a lesser extent dislocation climb in the grain boundary). As lattice peaks are removed at the leading edge (added at the trailing edge), mass flows toward (away from) these sites. In the outer grains this manifest as a small translation that opposes the direction of translation of the embedded grain. 


\subsection{Geometric Model}

The role of lattice continuity and the geometric reason why lattice translation occurs with grain shrinkage follows from an extension of the ideas of Cahn and Taylor[3], and is given in Fig. 2. As the embedded grain shrinks, the lattice planes in the outer grain extend (Fig. 2(b)). This would break the continuity of the lattice planes across the circular grain boundary. The lattice planes in the embedded grain can maintain continuity of the planes perpendicular to the planar boundary if the embedded grain is translated parallel to the planar interface (Fig.2Fig.2(c)). However, the continuity of the lattice planes in the embedded grain that are parallel to the planar interface cannot be maintained by a rigid body motion and must be accommodated by strain. Near the leading edge, the lattice translation should lead to compressive strains in the embedded grain as the lattice planes are squeezed together and tensile strains in the outer grains as the lattice planes are pulled apart. Near the trailing edge the expected strains are reversed. If the continuity of the lattice planes across the boundary was never broken, these strains would increase to unrealistic values as the grain shrank. However, these strains are partially relieved by motion of the grain boundary dislocations tangentially to the grain boundary, since the motion of a dislocation can change which lattice planes extend from the outer grain to the embedded grain thus allowing for a better match of the lattice planes between the embedded and outer grains. 
Fig.2.(a) Schematic of the lattice plane continuity. (b) As the grain shrinks the lattice planes in the outer grains extend and are no longer continuous across the embedded grain boundary. (c) To recover the continuity of the lattice planes not parallel to the planar interface, the embedded grain translates. The continuity of the lattice planes parallel to the planar interface cannot be restored by a rigid body motion of the lattice and must be accommodated by strain.

To compare the strain in the simulations with those that follow from the proposed geometric model, the spatial distribution of dilatational strain2is given in Fig. 3. The embedded grain is translating from right to left. Fig. 3 shows that the dislocations that comprise the boundary induce compressive and tensile strains on the scale of their Burgers vector. Of more interest are the smaller compressive dilatational stains that occur in the leading edge of the embedded grain and in the outer grains near the trijunction at the trailing edge, in agreement with the geometrical picture of grain translation given in Fig. 2. Consistent with our proposed mechanism, tensile dilatational strains are present near the trailing edge of the embedded grain and near the leading edge trijunction ofthe outer grains.

Fig.3 (color online). Dilatational strain field.Note the compressive strains in the leading edge of the embedded grain and outside the trailing edge. Likewise the tensile strains in the trailing edge of the embedded grain and outside the leading edge.

\subsection{Dislocation Mechanism}

Since the displacement of the embedded grain is on the order of several lattice parameters, it cannot be accommodated by elastic strain. Thus, there must be

\footnotetext{
2 Strains were calculated using a finite element representation of the strain, which compares the peak positions to those of an unstrained lattice.The strain is calculated using the constant strain triangle[37] where the strain is assumed to be constant between lattice positions[38].
} 
another mechanism that allows the rigid body motion of the grain. We find that the grain boundary dislocations move via a combination of glide and climb, as shown in Fig. 4 for a dislocation on the leading edge, which allows the dislocations to move roughly perpendicular to the planar interface instead of along the close-packed direction of the Burgers vector (see Fig. 1). The climb mechanism also destroys lattice sites at the leading edge of the grain to allow for the rigid body translation. While the dislocation motion on the trailing edge is essentially the same, the climb is in the opposite direction thus creating lattice sites. This also follows the strain fields in the embedded grain. The compressive strains on the leading edge build up and are relieved by the removal of a lattice site of the embedded grain which moves the lattice towards the leading edge trijunction. Likewise, tensile strains build up on the trailing edge and are relieved by the addition of a lattice site which moves the lattice away from the trailing edge trijunction.Lattice sites are also created on the leading edge and destroyed on the trailing edge by dislocation interactions that occur at the trijunctions. These reactions effect the embedded grain in the same manner as dislocation climb but to a much larger extent as will be shown in Section 3.5. This illustrates that grain growth processes can depend on atomic mechanisms that occur not in the grain boundary, but in the bulk crystal. Thus, grain growth rate can be a function of the bulk diffusivity, and not simply the grain boundary mobility.

A critical condition that allows this process to take place is the availability of climb as a mechanism for dislocation motion.This requires vacancies to diffuse toward the dislocations on the leading edge and away from the dislocations on the trailing edge. 
Vacancies in the PFC model are non-localized and manifest as variations in the peak height (i.e. a larger vacancy concentration equals alower average atomic density)[25]. The non-local property of vacancies in this model makes it difficult to observe the process of vacancy diffusion.Furthermore, the value of the vacancy concentration is dependent on the parameterization for the non-dimensional form of the PFC used here. As an example, using the definition of vacancy density from Chan [33] and the parameterization for body-centered cubic iron [31,34], the variation in the vacancy density is 1.6 times the variation in the coarse grained density (see Appendix B of [35]). Fluctuations of the coarse grained density in the embedded grain are on the order of $10^{-4}$ giving variations in the vacancy density of the same order. The pattern of these fluctuations is complex and varies over time. It is not a simple gradient from the trailing to leading edge like the strain field shown in Fig. 3. Therefore, while the observed dislocation climb requires diffusion of vacancies to and from the dislocations, the sources and sinks of vacancies that are required for the climb is unclear and remains a topic for future study.

Fig.4 (color online). (a) System overview showing the position of (b)-(f). Following the motion of a single dislocation at times (b) 17,500, (c) 25,000, (d) 30,000, (e) 37,500, and (f) 42,500. The arrow overlaid on the density field indicates the Burgers vector and the two lines extending from it mark the extra half planes that comprise a perfect dislocation in this hexagonal lattice[36]. The black dots mark the peak locations. Moving from (b) to (c) the dislocation climbs which removes a lattice site (indicated by the (b) dashed then (c) solid black circle). Then from (c) to (d) it glides. From (d) to (e) it climbs again, and from (e) to (f) it glides again. Grain translation can be observed on the right edge of each image near the bottom indicated by the arrow. The peak starts partially cut off but becomes fully visible as time progresses.

To confirm that dislocation climb fully compensates for the translation of the lattice, the amount of translation predicted by coupling is compared to the number of 
lattice sites added or removed by dislocation climb. From Cahn and Taylor[3] for the case of pure coupling (no sliding) in small angle tilt boundaries, the tangential velocity of the lattice $\left(v_{t}\right)$ is proportional to the normal velocity of the grain boundary $\left(v_{n}\right)$ by the misorientation angle $(\theta)$ (Eq. 3).

$$
v_{t}=\theta v_{n},
$$

Integrating $v_{t}$ from the initial time until the embedded grain disappears yields the total displacement, $\delta$.Integrating $v_{n}$ over the same time gives the total distance the grain boundary travels which is simply the initial radius, $R_{0}$. The result, which is given in Eq. 4, is that the total amount of translation depends only on the misorientation and initial radius.

$$
\delta=-\theta R_{0},
$$

Since the misorientation sets the dislocation density and the initial radius sets the length of the boundary, the amount of translation is directly related to the dislocation content of the boundary. The Burgers vectors of the grain boundary dislocations can be grouped into two sets: those with Burgers vectors that are parallel to the direction of translation and those that are not. The dislocation shown in Fig. 4 is an example of a dislocation with a Burgers vector that is not parallel to the translation direction, andthese dislocations climb every other lattice plane. The dislocations with Burgers vectors parallel to the translation direction must climb every lattice plane because the Burgers vector is perpendicular to the direction the dislocation is climbing (see Fig. 1). The component of the Burger's vector in the direction of translation (i.e. the $x$ direction) has a magnitude of 1 for the dislocations with Burgers vectors parallel to the translation direction while the other 
dislocations have a magnitude of $1 / 2$, in units of the lattice parameter. The number of lattice sites added (or removed) per dislocation then corresponds to the component of the Burgers vector in the translation direction. Following the work of $\mathrm{Wu}$ and Voorhees[16], the net Burgers vector content $\left(\vec{n}_{b}\right)$ of a segment $(\vec{r})$ of a planar lowangle grain boundary with two sets of dislocations can be written as

$$
\vec{n}_{b}=N_{1} \vec{b}_{1}+N_{2} \vec{b}_{2}=r \theta \hat{n},
$$

where $\theta$ is the misorientation between the grains, $\hat{n}$ is the interface normal, and $r=|\vec{r}|$ is the length of the interface. Assuming a perfectly circular grain as the initial condition, and infinitesimal grain boundary segment can be described as $d r=R d \Phi$, where $R$ is the radius and $\Phi$ is the polar angle. The normal vector to $d r$ is $\hat{n}=-\cos \Phi \hat{x}-\sin \Phi \hat{y}$.The net Burgers vector content of a section of interface can then be written as

$$
d \vec{n}_{b}=-R \theta(\cos \Phi \hat{x}+\sin \Phi \hat{y}) d \Phi
$$

By symmetry, the $x$ component of the net Burgers vector for the grain boundary between the embedded grain and upper grain is the same as the grain boundary between the embedded grain and lower grain. Likewise, the net Burgers vector of the leading edge is equal and opposite to the that of the trailing edge because on the leading edge lattice are removed and on the trailing edge lattice sites are added. Therefore, the amount of translation due to dislocation climb can be calculated by integrating Eq. 6 over a quarter of the grain boundary (e.g. 0 to $\pi / 2$ ). The $x$ component of the result is identical to Eq. 4 showing that the translation due to coupling is fully allowed by climb of dislocations in the interface.

\subsection{The Effects of Trijunctions}


While climb of the dislocations in the boundary facilitates and is required for the translation process, reactions of dislocations at the trijunctions are needed for this process to move forward, since the only place dislocations are removed is at the trijunctions, and the total length of the upper and lower grain boundaries decreases with time. The chemical potential $\left(\mu=\frac{\delta \mathcal{F}}{\delta \psi}\right)$ of the system is one measure of the trijunctions' impact (since the chemical potential varies with the density field, it is coarse grained to produce the local average). As a grain boundary dislocation approaches the dislocation at a trijunction, a large positive peak in the chemical potential develops near the trijunction that is up to an order of magnitude larger than the variations in the chemical potential at other times. A convenient method for tracking when these peaks occur is to calculate the range of the chemical potential at each time step which we define as $\mu_{\text {range }}=\mu_{\max }-\mu_{\min } \cdot \mu_{\max }$ is the maximum value of the chemical potential, and $\mu_{\min }$ is minimum valueover the entire system. Large values of $\mu_{\text {range }}$ only occur at times corresponding to the trijunction reactions (see Fig. 5). The trijunction reactions can be generally classified into two categories: reactions that bend the grain boundary between the matrix grains out of plane and reactions that restore that grain boundary to planar. Both reactions accelerate the embedded grain's translation, but the reactions that restore the grain boundary to planarity have significantly more effect. In the interval between the trijunction reactions, the velocity of the embedded grain moves toward zero until another trijunction reaction occurs. 
The dislocation reactions at the trijunction also create large strains in the vicinity of the trijunction. As a dislocation approaches the trijunction, there is an attractive force between the dislocation in the boundary and the one in the trijunction due to the strain fields generated by the dislocations. As the dislocation is pulled into the trijunction, the local dislocation spacing increases and becomes inconsistent with the dislocation separation that is given by the Frank formula, or the misorientation. Since neither grain can rotate, large strains develop (Fig. 6) which are subsequently relieved by motion of the grain boundary (shrinking) and the corresponding translation.

Fig. 5 (color online). Effect of the trijunctions on grain translation. (a) The large spikes in the range of the chemical potential (in pairs for the two trijunctions, left and right, in the system) correspond to dislocation reactions at the trijunctions. These reactions result in an increase in the magnitude of the velocity of the embedded grain, thus enabling the grain translation process. The structure of the left trijunction is shown at times (b) 1,050,000 (c) 1,250,000 and (d) 1,500,000. From (b) to (c) the grain boundary between the matrix grains is bent out of plane due to the dislocation reaction. The resulting velocity increase is relatively small. From (c) to (d), the dislocation reaction at the trijunction restores the boundary to planar, and the resulting increase in velocity is significant.

Fig.6 (color online).The dilatational strain field (a \& b) and the local orientation (c \& d) at times 997,500 (a \& c) and 1,002,500 (b \& d). Large strains develop near the trijunction immediately following the trijunction reaction along with an area of intermediate orientation. These develop due to a mismatch between the equilibrium dislocation spacing for the misorientation and the actual dislocation spacing following the reaction. The arrows show the Burgers vectors of the dislocations with the gray arrows indicating the dislocations involved in the reaction.

\subsection{Trijunctions at Lower Temperatures}

The importance of trijunctions increases at lower temperatures(i.e. larger $\epsilon$ ) and further from the phase boundary ( $\bar{\psi}$ closer to 0$)$. At very low temperatures grain growth eventually stops when climb is no longer feasible. However, the embedded grain can stop shrinking above this temperature, while a circular grain 
embedded in a single crystal is still able to shrink[29].Since the grain embedded in a single crystal requires dislocation climb to shrink, the arrest of grain growth in this intermediate temperature regime is not due to the difficulty of dislocation climb, but is linked to the dislocation reactions that occur at the trijunction.

As a dislocation in the boundary moves toward the trijunction,its ability to react with the dislocation in the trijunction determines whether grain growth will continue or stall. Globally, the dislocation is pushed towards the trijunction by the curvature of the grain boundary. Locally, the stain fields of the two dislocations with appropriate Burger's vectors, can provide a larger attractive force. If the Burgers vectors are $120^{\circ}$ apart,the dislocations each contain a half plane that can combine to form a full plane and a single dislocation with a Burgers vector that is $60^{\circ}$ from both original dislocations. As the dislocation approaches the trijunction, its slip plane is not usually aligned with the slip plane of the dislocation in the trijunction such that they can combine via glide, and climb is necessary to align them. It is only when the slip planes of the two dislocation align that the large attractive force is present to the boundary dislocation into the trijunction. Opposing this attractive force is the strain created when the dislocation moves into the boundary. While in general the dislocations in the grain boundary of the embedded grain move uniformly to preserve the dislocation spacing, when the dislocation is pulled into the trijunction the local dislocation spacing changes from the equilibrium spacing expected from the misorientation angle. This creates an area of smaller misorientation and large strains in the trijunction (Fig. 6). 
The ability of dislocations to pass through each other is not necessary for trijunction reactions, but greatly facilitates it. The planar grain boundary that is formed at the trijunction as the embedded grain shrinks is composed of roughly alternating dislocations with Burgers vectors that are $60^{\circ}$ apart (this pairing can be seen at the end of the dislocation paths in Fig. 1). If dislocations can pass through one another, they do not have to arrive at the trijunction with alternating Burgers vectors to maintain a flat boundary. While the grain can continue to shrink even with some roughness to the planar boundary (e.g. see Fig. 5), significant deviation of the boundary requires dislocations on the upper and lower side of the embedded grain to travel different distances to reach the trijunction. Since the motion of these dislocations is coupled to the translation of the lattice, this requires that the upper and lower parts of the grain translate different distances. The additional strain associated with this can also impede the dislocation reactions at the trijunctions.

At lower temperatures, it is well known that the elastic constants increase and thus the elastic strain energy increases. This increase in elastic energy provides more opposition to the dislocation reaction at the trijunction than at higher temperatures. This is evident in the energy curves shown in Fig. 7 (a). The drops in energy correspond to dislocation reactions at the trijunctions and the height of those drops increases with $\epsilon$. By $\epsilon=0.13$, grain growth stalls after just one trijunction reaction. More importantly, the slope of the energy curve before the trijunction reaction approaches zero as $\epsilon$ increases. The system has now reached a metastable 
equilibrium point where motion of the dislocation toward the trijunction would cause the energy of the system to increase, despite the decrease in energy associated with decreasing the grain boundary length of the embedded grain. The chemical potential of the system is uniform after growth is stalled (Fig. 7 (c)) compared to a higher chemical potential in the embedded grain during growth (Fig. 7 (b)). The addition of noise (as normally distributed random fluctuations added to the density field) did not restart grain growth, even in the extreme case of noise with a standard deviation of 0.5 (as compared to the density range from minimum to maximum of 0.8 ), indicating that this metastable state is extremely stable.In addition, if the metastable equilibrium condition in Fig. $7(\mathrm{c})$ is used as an initial condition at slightly higher temperature the grain will shrink, indicating that when the elastic stress present at lower temperature decreases there is no longer an impendent to grain shrinkage.

\subsection{High-angle Grain Boundaries}

The three-grain system was also examined with a misorientation of $15.2^{\circ}$ to observe any differences in behavior compared to the low-angle system. At $\epsilon=0.10$ the grain boundary is wet and no coupling is observed. At $\epsilon=0.12$ the behavior is similar to the low-angle case albeit with slower dynamics. Using the same analysis, the coupling parameter is about $1 \%$ less than expected by theory. At $\epsilon=0.13$, grain growth is stalled just as it is in the low-angle case. The primary difference between low- and high-angle grain boundaries in this system is the lack of coupling at higher 
temperatures as the grain boundary becomes wet and the lattice is no longer

continuous across the boundary.

Fig.7 (color online). (a)Evolution of the energy of the system relative to the equilibrium bicrystal for an embedded grain with an initial radius of 31.7 and $\epsilon$ of $0.10,0.12$, and 0.13 . In the case where $\epsilon=0.13$ growth is stalled. The significant drops in the energy correspond to dislocation reactions at the trijunctions, and the magnitude of the drop increases with $\epsilon$. The local average chemical potential referenced to the system average chemical potential is shown for $\epsilon=0.13$ at times (b) 72,500 and (c) $\mathbf{7 5 0 , 0 0 0}$ with the black dots representing the dislocation positions. (b) The chemical potential is higher in the embedded grain, and there is a local variation near where a climb event occurred. (c) The chemical potential is uniform after growth is stalled indicating a metastable equilibrium.

Fig.8 (color online). Local average density fields of the multigrain simulations at times (a-c) 500,000 and (d-f) 1,500,000 for $\epsilon$ of $(a, d) 0.10$, (b, e) 0.12 , and (c, f) 0.13 . The lower average density occurs around dislocations and grain boundaries.

\subsection{Multigrain Simulations}

To observe grain translation in a more complex system, a square domain of size $3214^{2}$ at $\epsilon=0.12$ was seeded with random density fluctuations and evolved until grains formed. The resulting microstructure was then coarsened at $\epsilon$ of 0.10 , 0.12 , and 0.13 (See Fig. 8).At $\epsilon=0.10$, grain growth is dominated by high angle grain boundaries which move by sliding (as discussed in Section 3.7) and have a significantly higher mobility. At $\epsilon=0.12$, the high angle grain boundaries also exhibit coupling, and translation can be seen during coarsening. Fig. 9 shows the evolution of one grain in the system as it shrinks. There is little change in the grains orientation $\left(\sim 1^{\circ}\right)$, and the paths of the peaks in the grain show translation as expected. Analysis of other grains in the system indicates that while the case of pure translation may not commonly occur(only3 cases out of over 150 grains), grains often exhibit some combination of rotation and translation.Further study is needed to quantify the effects of translation in complex polycrystalline systems. 
Fig.9 (color online). The local orientation of a grain from the multigrain simulation at $\epsilon=0.12$. (a) Grain at time 112,500 with the black dots representing some of the peak locations. (b) At time 715,000 the grain has shrunk with little change in its orientation $\left(\sim 1^{\circ}\right)$. The black lines represent the paths the peaks took and shows translation.

\subsection{The Effect of Dimensionality and Crystal Symmetry}

The results presented here are all in two-dimensions (2D) and for the hexagonal crystal structure. Remaining in 2D, a different crystal structure, such as square, will change the dislocation structure of the grain boundary. While this affects the ease at which dislocations move and the reactions that occur at thetrijunctions, the basic geometric description of the translation process shown in Fig. 2 is not dependent on the crystal structure. As long as the lattice planes are continuous across the grain boundary and the dislocations are able to climb, translation should occur in all 2D crystal structures for some range of temperatures.

The transition from 2D to three-dimensions (3D) will have a larger impact than changing the crystal structure. Attempting to translate the geometric model into 3D presents significant complications because the lattice plane continuity across the grain boundary is more complex. For the case of a cylindrical grain embedded at a symmetric tilt boundary, the behavior in 3D should be similar to the 2D case presented here, butthe extra degree of freedom allows the orientation of the embedded grain to vary in more ways that would not necessarily match this model. For the case of a spherical grain embedded at a symmetric tilt boundary, the dislocation structure is significantly more complex, and the simple model used here no longer captures all the continuous lattice planes. Further study is needed to understand the effects of coupling in 3D. 


\section{Conclusion}

In conclusion, the continuity of lattice planes across a grain boundary can give rise to grain translation in polycrystalline systems during grain growth. We show that as a grain that is embedded in a bicrystal boundary shrinks, grain boundary dislocations climb to add or remove lattice sites on the trailing and leading edge of the grain which allows translation to occur. When the dislocations approach the trijunction, they may react, thus enabling grain translation and allowing the embedded to continue shrinking. At intermediate temperatures the ability of the embedded grain to translate and shrink is limited by dislocation reactions at the trijunctions. At low temperatures grain growth arrests if these reactions do not occur. The presence of lattice translation in the large multigrain simulations demonstrates that this phenomenon is not limited to an isolated three grain system.No size dependence was observed in our calculations over the range of grain sizes from 60 to 120 lattice parameters in diameter, whether this continues to larger sizes is a question for investigation.

\section{Acknowledgements}

This work was supported by the National Science Foundation through grant Nos. DMR 1105409 and 1507033, the National Science Council of Taiwan (NSC102-2112M-007-007-MY3), and the National Center for Theoretical Sciences, Taiwan.

\section{References}

[1] H. Thomas, G.E. Morfill, V. Demmel, J. Goree, B. Feuerbacher, D. Möhlmann, Plasma Crystal: Coulomb Crystallization in a Dusty Plasma, Phys. Rev. Lett. 73 
(1994) 652-655. doi:10.1103/PhysRevLett.73.652.

[2] L. Ramos, T.C. Lubensky, N. Dan, P. Nelson, D.A. Weitz, Surfactant-Mediated Two-Dimensional Crystallization of Colloidal Crystals, Science (80-. ). 286 (1999) 2325-2328. doi:10.1126/science.286.5448.2325.

[3] J.W. Cahn, J.E. Taylor, A unified approach to motion of grain boundaries, relative tangential translation along grain boundaries, and grain rotation, Acta Mater. 52 (2004) 4887-4898. doi:10.1016/j.actamat.2004.02.048.

[4] J.W. Cahn, Y. Mishin, A. Suzuki, Coupling grain boundary motion to shear deformation, Acta Mater. 54 (2006) 4953-4975. doi:10.1016/j.actamat.2006.08.004.

[5] V.A. Ivanov, Y. Mishin, Dynamics of grain boundary motion coupled to shear deformation: An analytical model and its verification by molecular dynamics, Phys. Rev. B - Condens. Matter Mater. Phys. 78 (2008) 1-12. doi:10.1103/PhysRevB.78.064106.

[6] T. Gorkaya, D.A. Molodov, G. Gottstein, Stress-driven migration of symmetrical

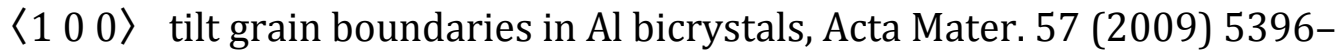
5405. doi:10.1016/j.actamat.2009.07.036.

[7] D.L. Olmsted, E.A. Holm, S.M. Foiles, Survey of computed grain boundary properties in face-centered cubic metals-II: Grain boundary mobility, Acta Mater. 57 (2009) 3704-3713. doi:10.1016/j.actamat.2009.04.015.

[8] D.A. Molodov, T. Gorkaya, G. Gottstein, Dynamics of grain boundaries under applied mechanical stress, J. Mater. Sci. 46 (2011) 4318-4326. doi:10.1007/s10853-010-5233-6.

[9] Z.T. Trautt, A. Adland, A. Karma, Y. Mishin, Coupled motion of asymmetrical tilt grain boundaries: Molecular dynamics and phase field crystal simulations, Acta Mater. 60 (2012) 6528-6546. doi:10.1016/j.actamat.2012.08.018.

[10] A. Karma, Z.T. Trautt, Y. Mishin, Relationship between equilibrium fluctuations and shear-coupled motion of grain boundaries, Phys. Rev. Lett. 109 (2012) 1-5. doi:10.1103/PhysRevLett.109.095501.

[11] A. Rajabzadeh, F. Mompiou, S. Lartigue-Korinek, N. Combe, M. Legros, D.A. Molodov, The role of disconnections in deformation-coupled grain boundary migration, Acta Mater. 77 (2014) 223-235. doi:10.1016/j.actamat.2014.05.062.

[12] T.J. Rupert, D.S. Gianola, Y. Gan, K.J. Hemker, Experimental observations of stress-driven grain boundary migration., Science. 326 (2009) 1686-1690. doi:10.1126/science.1178226.

[13] J.A. Sharon, P.C. Su, F.B. Prinz, K.J. Hemker, Stress-driven grain growth in nanocrystalline Pt thin films, Scr. Mater. 64 (2011) 25-28. doi:10.1016/j.scriptamat.2010.08.057. 
[14] M. Winning, A.D. Rollett, G. Gottstein, D.J. Srolovitz, A. Lim, L.S. Shvindlerman, Mobility of low-angle grain boundaries in pure metals, Philos. Mag. 90 (2010) 3107-3128. doi:10.1080/14786435.2010.481272.

[15] A.T. Lim, M. Haataja, W. Cai, D.J. Srolovitz, Stress-driven migration of simple low-angle mixed grain boundaries, Acta Mater. 60 (2012) 1395-1407. doi:10.1016/j.actamat.2011.11.032.

[16] K.-A. Wu, P.W. Voorhees, Phase field crystal simulations of nanocrystalline grain growth in two dimensions, Acta Mater. 60 (2012) 407-419. doi:10.1016/j.actamat.2011.09.035.

[17] A. Adland, Y. Xu, A. Karma, Unified Theoretical Framework for Polycrystalline Pattern Evolution, Phys. Rev. Lett. 110 (2013) 265504. doi:10.1103/PhysRevLett.110.265504.

[18] N. Bernstein, The influence of geometry on grain boundary motion and rotation, Acta Mater. 56 (2008) 1106-1113. doi:10.1016/j.actamat.2007.11.002.

[19] Z.T. Trautt, Y. Mishin, Grain boundary migration and grain rotation studied by molecular dynamics, Acta Mater. 60 (2012) 2407-2424. doi:10.1016/j.actamat.2012.01.008.

[20] S.G. Srinivasan, J.W. Cahn, Challenging Some Free-Energy Reduction Criteria for Grain Growth, in: S. Ankem, C.S. Pande, I. Ovid'ko, S. Ranganathan (Eds.), Sci. Technol. Interfaces, TMS, Seatle (WA), 2002: pp. 3-14.

[21] M. Upmanyu, D.J. Srolovitz, a. E. Lobkovsky, J. a. Warren, W.C. Carter, Simultaneous grain boundary migration and grain rotation, Acta Mater. 54 (2006) 1707-1719. doi:10.1016/j.actamat.2005.11.036.

[22] J. Berry, M. Grant, K. Elder, Diffusive atomistic dynamics of edge dislocations in two dimensions, Phys. Rev. E. 73 (2006) 31609. doi:10.1103/PhysRevE.73.031609.

[23] P. Stefanovic, M. Haataja, N. Provatas, Phase-field crystals with elastic interactions, Phys. Rev. Lett. 96 (2006) 1-4. doi:10.1103/PhysRevLett.96.225504.

[24] K. Elder, M. Katakowski, M. Haataja, M. Grant, Modeling Elasticity in Crystal Growth, Phys. Rev. Lett. 88 (2002) 245701. doi:10.1103/PhysRevLett.88.245701.

[25] K. Elder, M. Grant, Modeling elastic and plastic deformations in nonequilibrium processing using phase field crystals, Phys. Rev. E. 70 (2004) 20. doi:10.1103/PhysRevE.70.051605.

[26] Z.F. Huang, K.R. Elder, Mesoscopic and microscopic modeling of island formation in strained film epitaxy, Phys. Rev. Lett. 101 (2008) 1-4. doi:10.1103/PhysRevLett.101.158701. 
[27] K.-A. Wu, P. Voorhees, Stress-induced morphological instabilities at the nanoscale examined using the phase field crystal approach, Phys. Rev. B. 80 (2009) 125408. doi:10.1103/PhysRevB.80.125408.

[28] M. Bjerre, J.M. Tarp, L. Angheluta, J. Mathiesen, Rotation-induced grain growth and stagnation in phase-field crystal models, Phys. Rev. E - Stat. Nonlinear, Soft Matter Phys. 88 (2013) 1-4. doi:10.1103/PhysRevE.88.020401.

[29] R. Backofen, K. Barmak, K.E. Elder, A. Voigt, Capturing the complex physics behind universal grain size distributions in thin metallic films, Acta Mater. 64 (2014) 72-77. doi:10.1016/j.actamat.2013.11.034.

[30] J.M. Tarp, J. Mathiesen, Rotation-limited growth of three-dimensional bodycentered-cubic crystals, Phys. Rev. E. 92 (2015) 12409. doi:10.1103/PhysRevE.92.012409.

[31] K. Wu, A. Karma, Phase-field crystal modeling of equilibrium bcc-liquid interfaces, Phys. Rev. B. 76 (2007) 12. doi:10.1103/PhysRevB.76.184107.

[32] J. Mellenthin, A. Karma, M. Plapp, Phase-field crystal study of grain-boundary premelting, Phys. Rev. B. 78 (2008) 24. doi:10.1103/PhysRevB.78.184110.

[33] V.W.L. Chan, An In-Depth Examination of a Thermodynamic Framework for the Phase-Field Crystal Model, University of Michican, 2015.

[34] A. Jaatinen, C. Achim, K. Elder, T. Ala-Nissila, Thermodynamics of bcc metals in phase-field-crystal models, Phys. Rev. E. 80 (2009) 31602. doi:10.1103/PhysRevE.80.031602.

[35] K.S. Mcreynolds, Mechanisms in Grain Growth Studied with the Phase Field Crystal Model, Northwestern University, 2016.

[36] M.A. Meyers, K.K. Chawla, Mechanical Behavior of Materials, 2nd ed., Cambridge University Press, 2009.

[37] Z. Bittnar, J. Šejnoha, Numerical Methods in Structural Mechanics, ASCE Press, 1996.

[38] P.H. Mott, A.S. Argon, U.W. Suter, The atomic strain tensor, J. Comput. Phys. 101 (1992) 140-150. doi:10.1016/0021-9991(92)90048-4. 


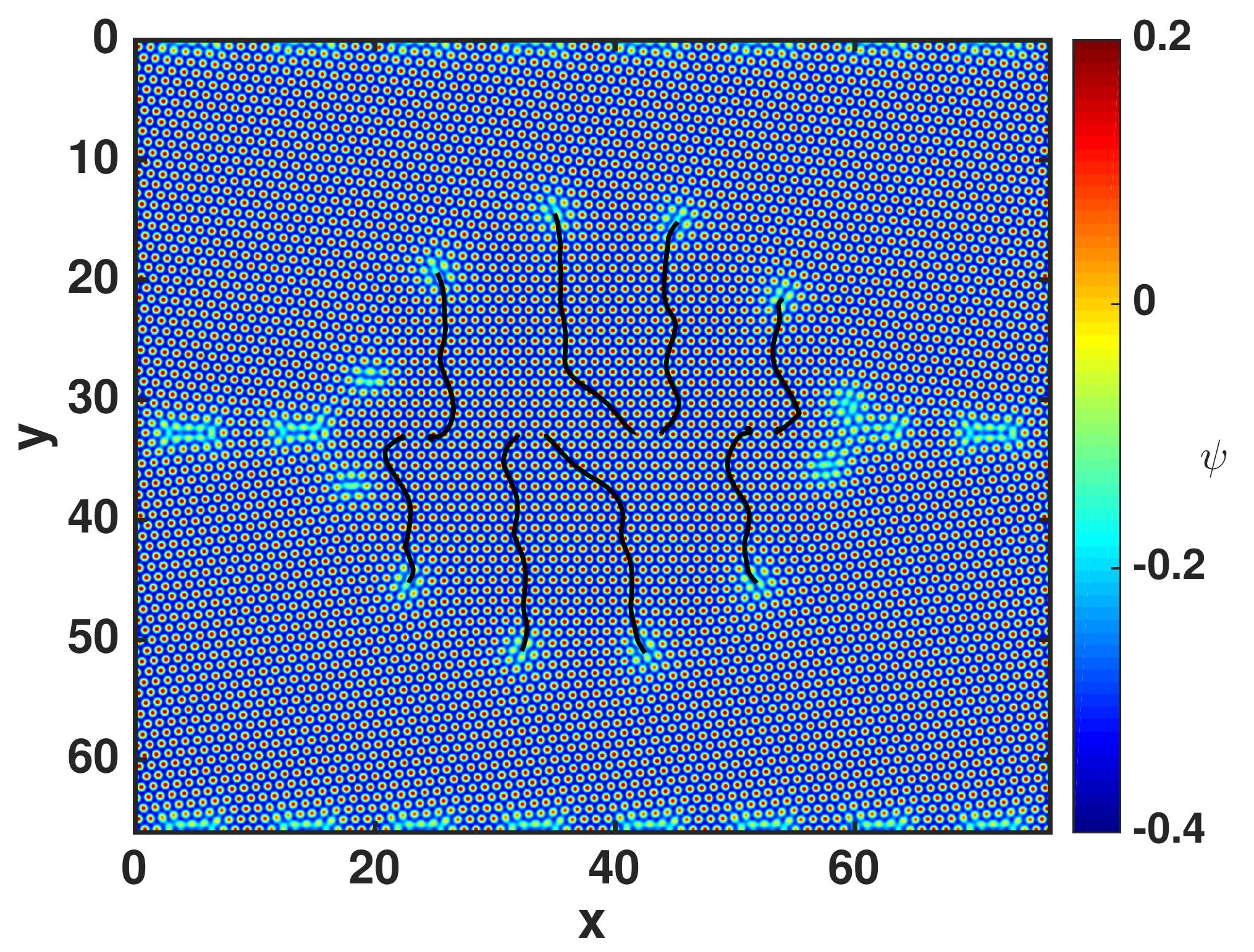




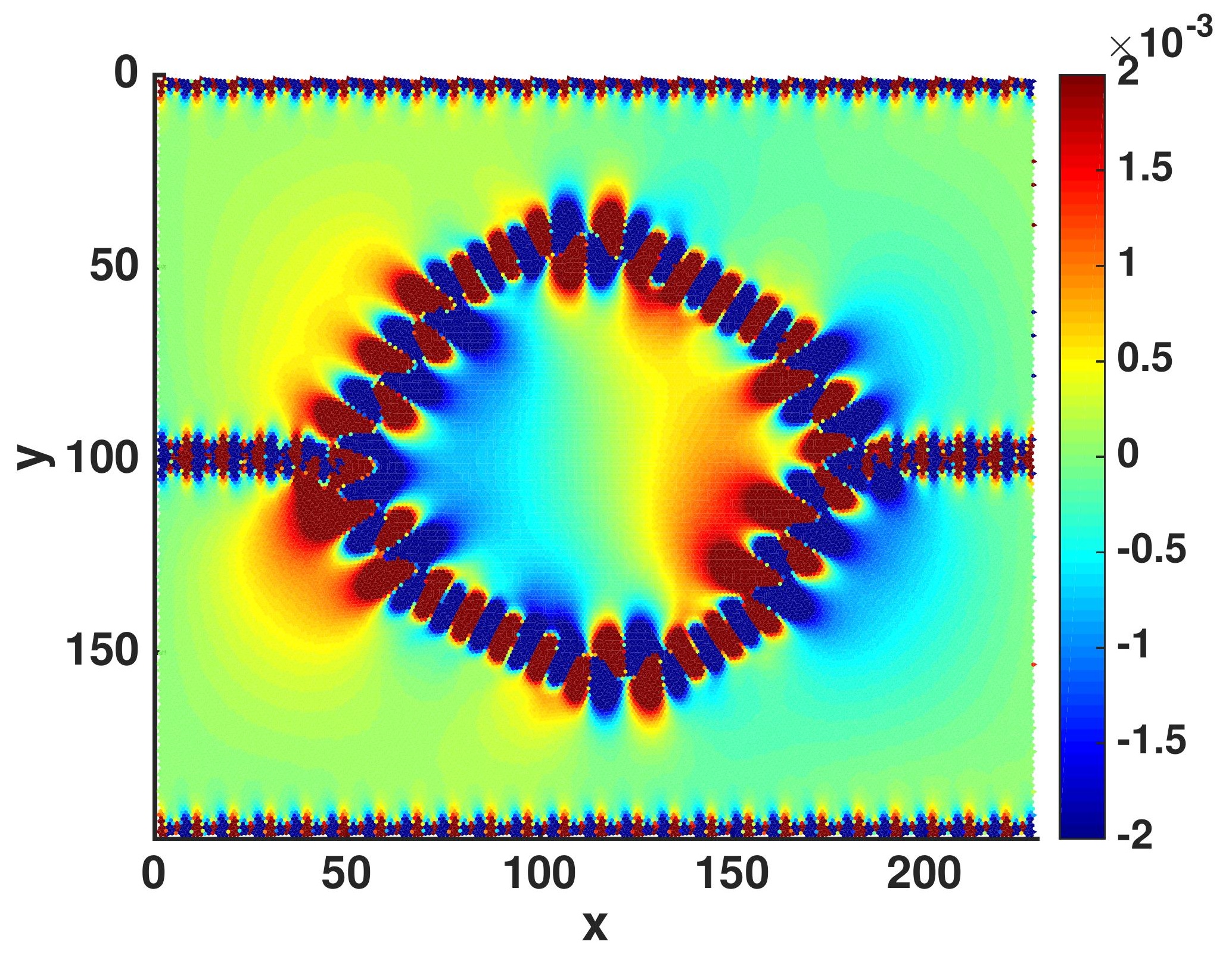



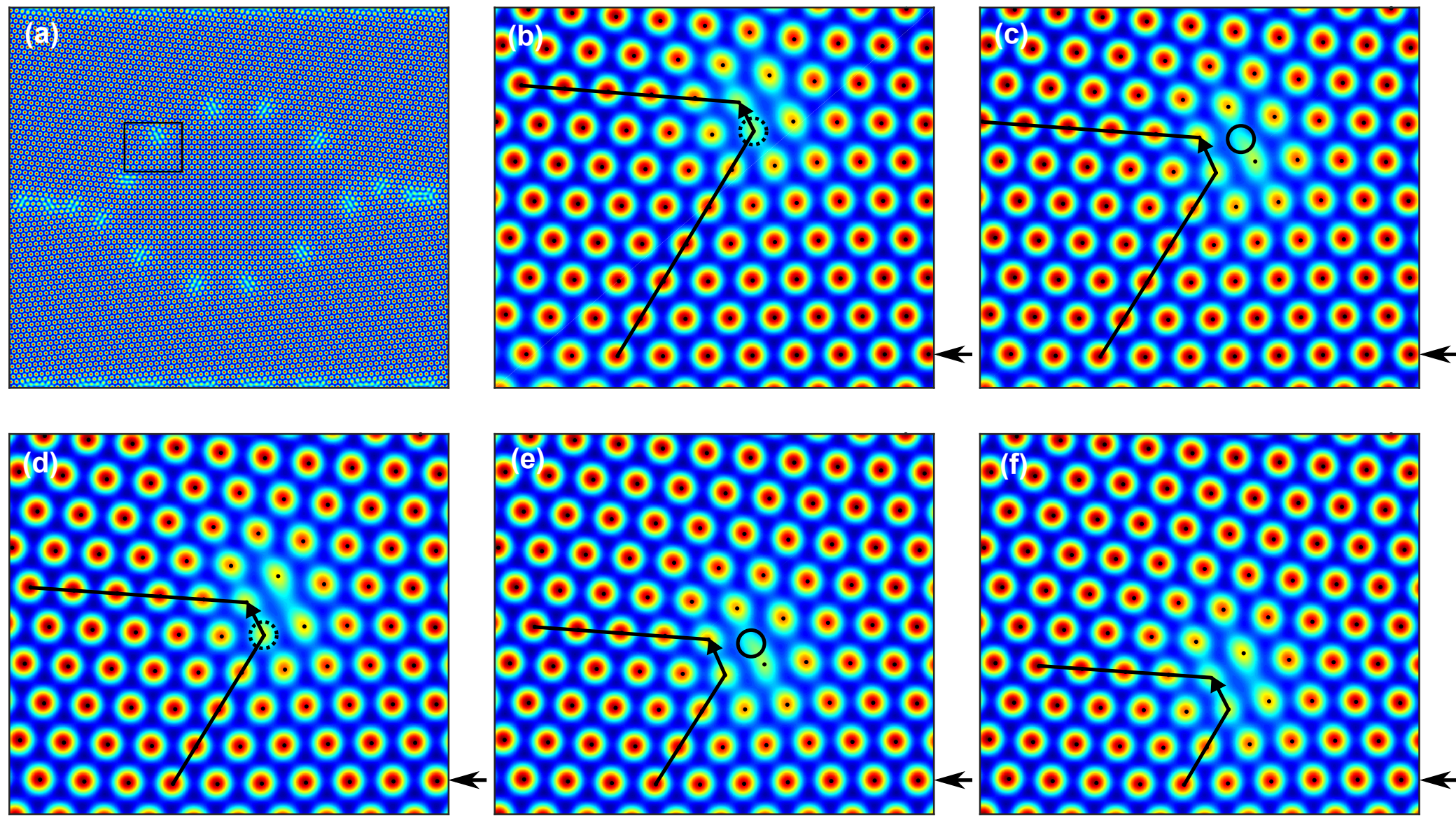


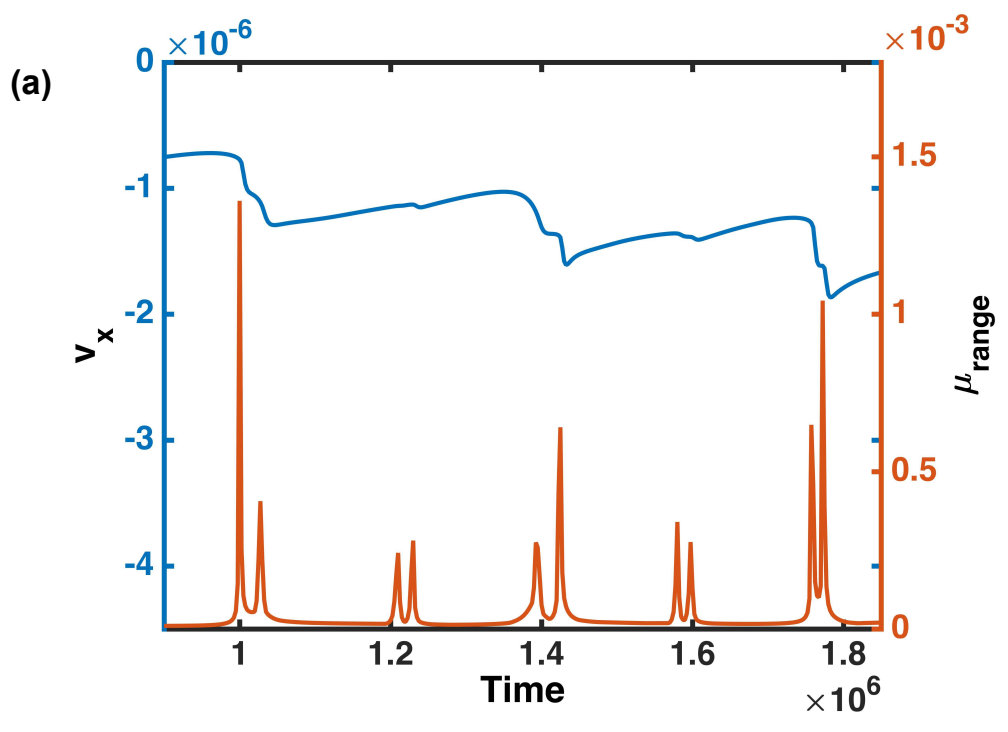

(b)

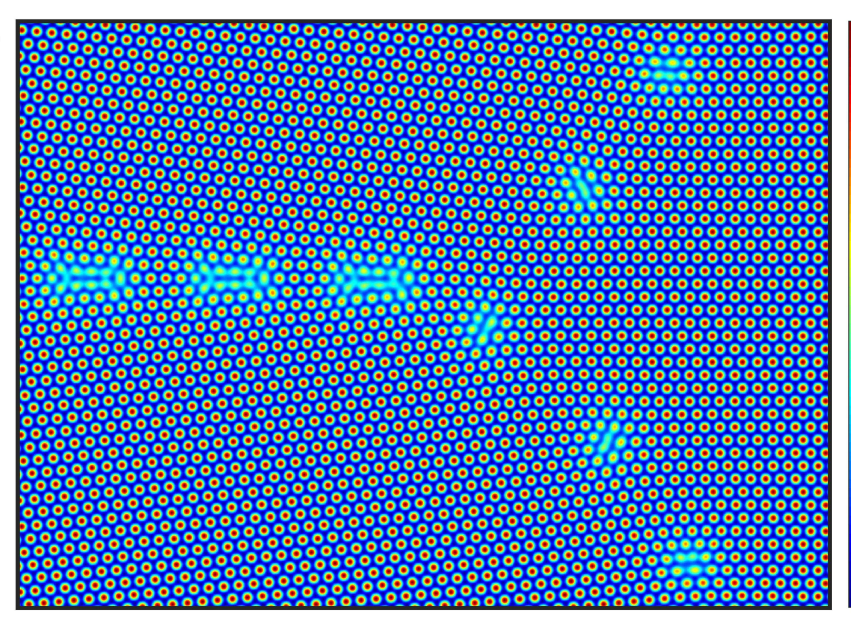

0.2

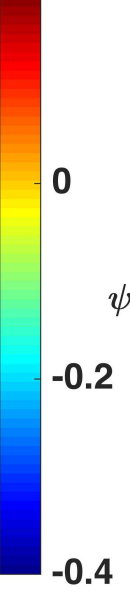

(c)

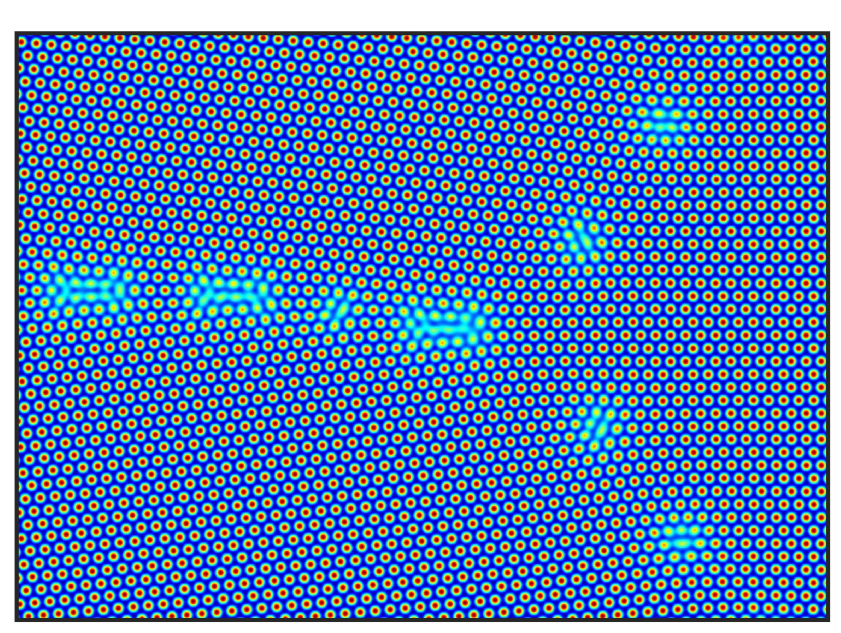

0.2

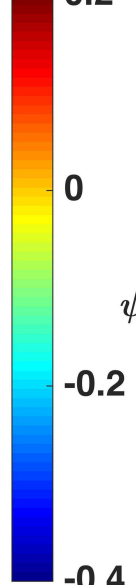

(d)

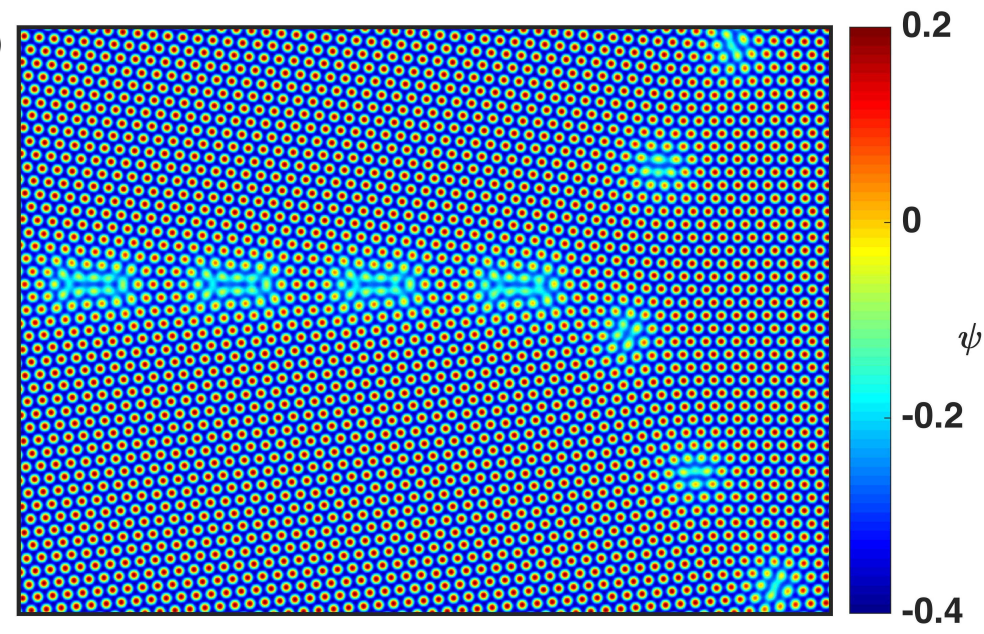


(a)

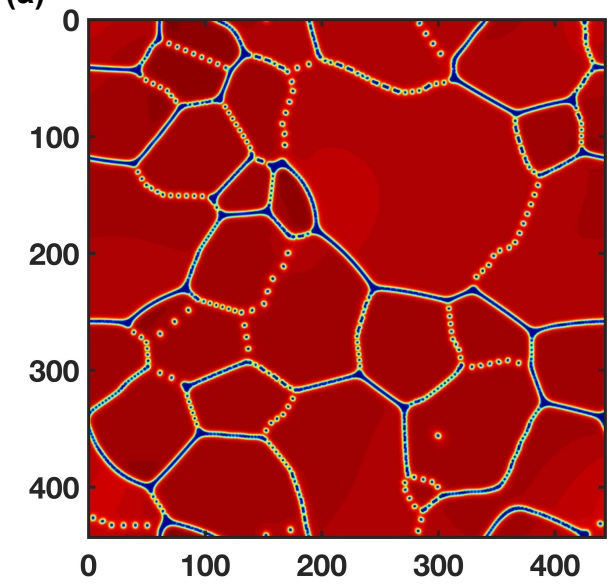

(d)

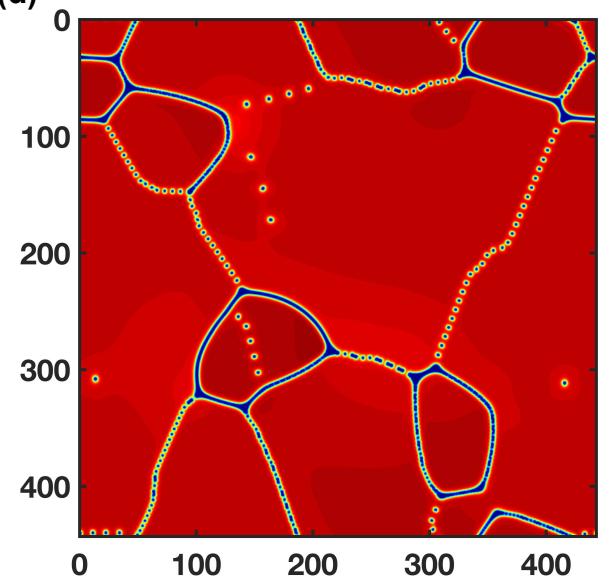

(b)

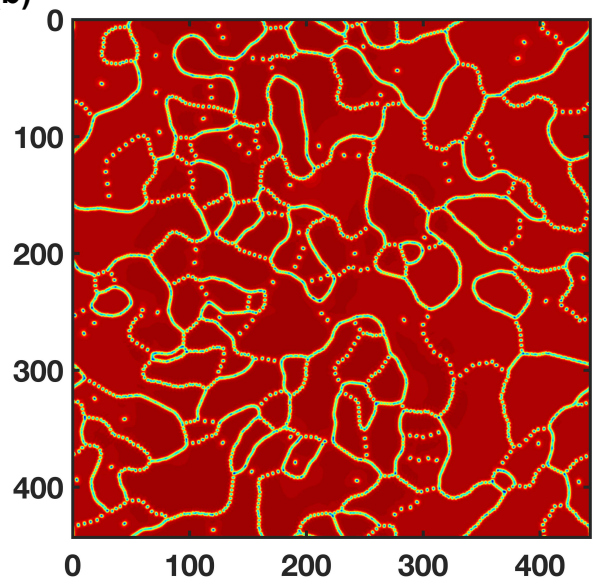

(e)

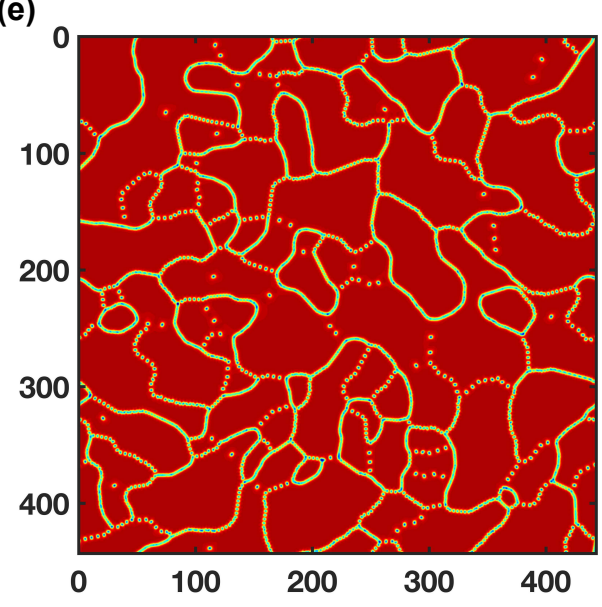

(c)

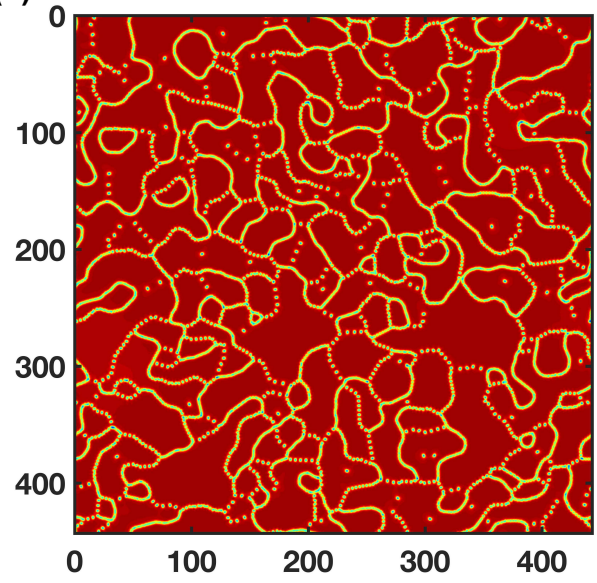

(f)

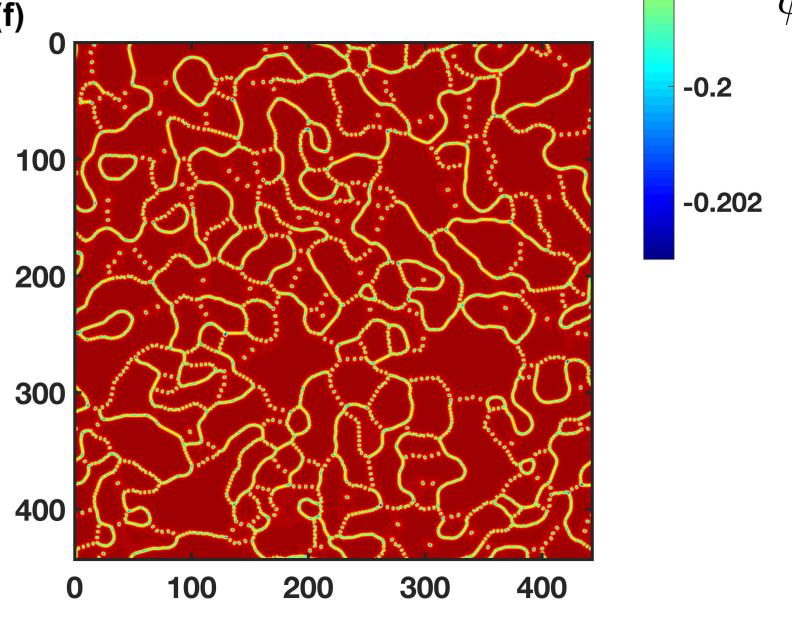


Figure 9

(a)

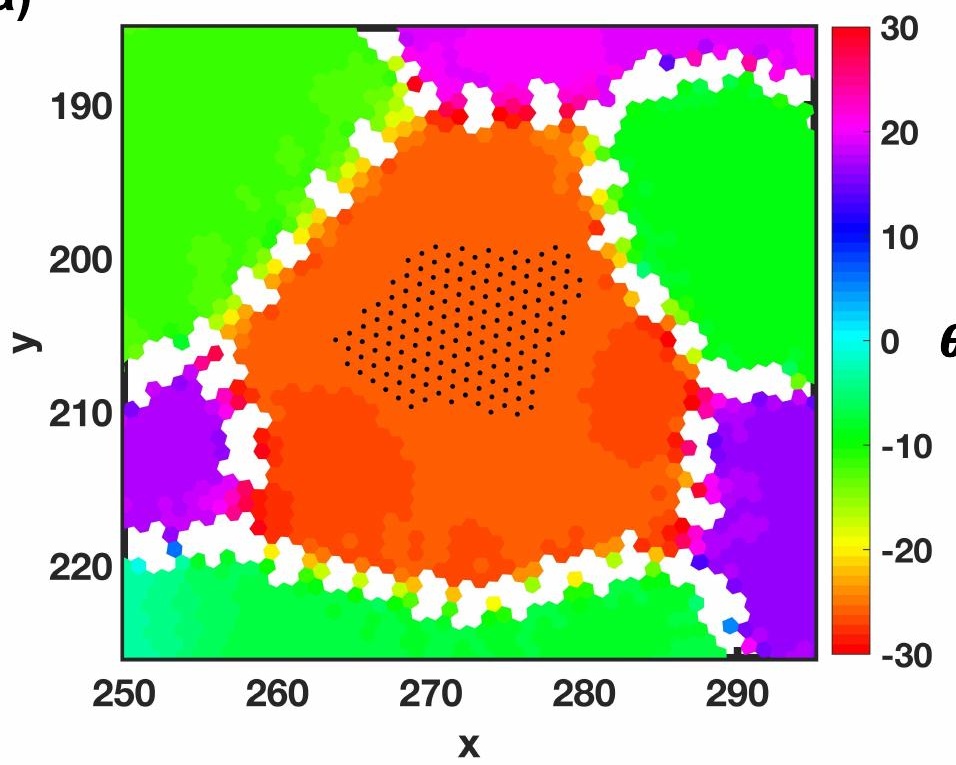

(b)

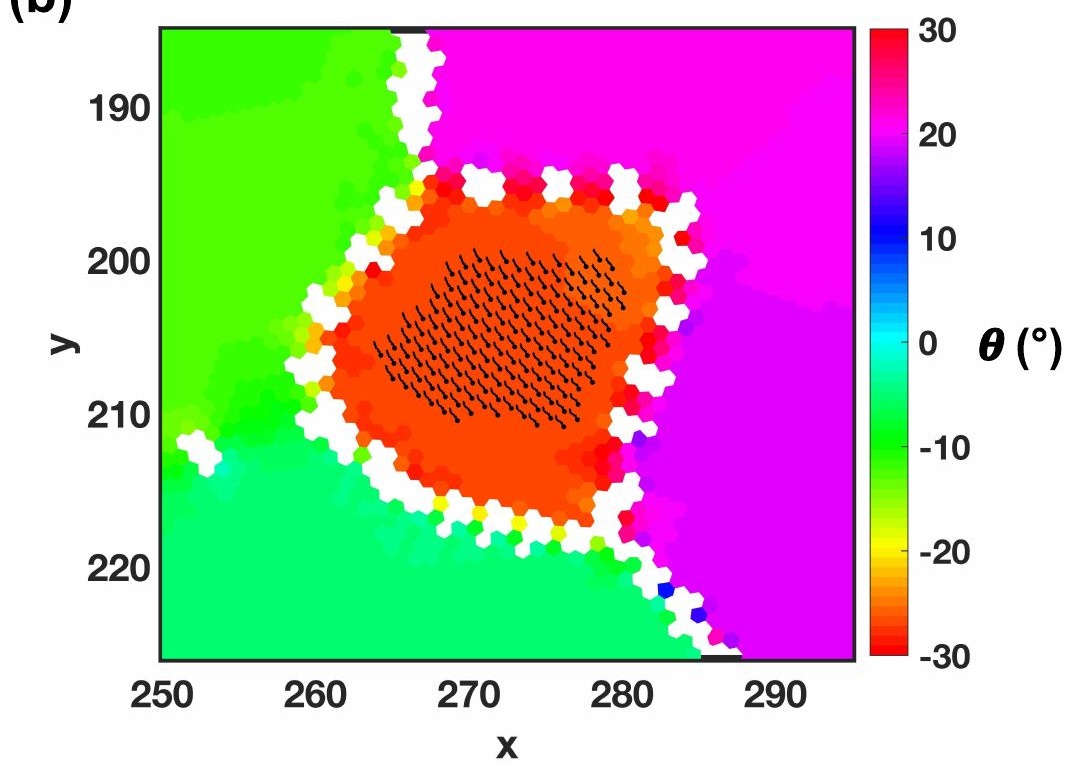


